We would be interested to hear the authors' response to these seeming inconsistencies.

N. E. DE TARANTo

A. Molivar

P. FLOWERS

J. SCOTT

E. ELSWORTH

M. JENKINS

M. Chapman

W. HOPKINS

D. TWENA

Department of Psychiatry

Chase Farm Hospital

Enfield, Middlesex

AUTHORS' REPLY: We are grateful to our colleagues at Chase Farm Hospital for pointing out the discrepancies in the Tables of our recent paper, and are flattered that the paper was chosen for such close scrutiny in what sounds like a stimulating Journal Club.

To answer the points:

(a) and (b) This was due to the erroneous transposition of one case (a heterosexual in the onset $<13$ group) from Tables 1 and 2 to the "unknown" category in Table 3.

(c) A proof error occurred in Table 2 and the column under core positive with regard to sexual arousal for cross-dressing should read 3, 30, 2 and not $0,33,2$. After this correction, Tables 2 and 3 then agree in that there were ten subjects with sexual arousal. The discrepancy between these tables and Table 1 is due to the definition used which was slightly different when assessing the DSM-III criteria and the presence of core transsexualism - in the former it was defined in terms of arousal with crossdressing (a narrower definition) whereby in the latter, a slightly wider definition was used to capture the sense of a total absence of sexual arousal in any aspect of the adoption of the cross-gender role.

(d) This was due to the addition of an "unknown" category to the "referral for surgery" in Table 2, whereas in Tables 1 and 3 these were included in the "not referred" group. To the best of our knowledge, these individuals were not referred for surgery.

While thanking Dr de Taranto and colleagues for bringing this to our attention, we feel these changes do not alter the main results or implications of our study.

Institute of Psychiatry

De Crespigny Park

London

\section{Musical and verbal hallucinations}

SIR: Berrios (Journal, February 1990, 156, 188-194) produced a useful literature review and statistical survey of cases of musical hallucinations. However, he does not seem to have read my related review of unilateral auditory hallucinations (Gordon, 1987) nor even my addendum in this journal (Gordon, 1988) drawing attention to it. Hence many interesting points were left unexplored and my conclusions were unchecked. Furthermore, it is now being cited as a 'definitive review' (Shapiro et al, 1991). To take some specific points:

Despite reporting personal cases with both musical and verbal hallucinations, Dr Berrios confined his review to musical ones only. Cases in the literature with otopathic auditory hallucinations cannot be clinically differentiated by type (i.e. music, words or elaborated tinnitus). Musical ones are admittedly quite striking given the specific and stirring quality of the evoked music. Indeed, it may not be coincidental that so many great composers had ear disease (Beethoven, Smetana, Schumann, etc). Nevertheless, most attention should be directed to non-musical auditory hallucinations, given their prominence in psychosis.

My references contained cases of musical hallucinations unused by $\mathrm{Dr}$ Berrios. There are undoubtedly others not cited by either of us.

I reported interesting relations between the laterality of the ear disease and hallucinations, but did not pursue this. Dr Berrios missed the chance to check this and did not even mention the subjective source of the hallucinations in any of his six new case vignettes.

My claim that hallucinations were more closely related to middle rather than inner ear deafness and to serous rather than purulent otitis was not assessed.

Dr Berrios made no distinction between unilateral and bilateral hallucinations. In medicine, causes of unilateral and bilateral diseases are often quite different. Focusing, as I did, on unilateral hallucinations simplifies correlation with ear or brain disease. Although I do not doubt that ear disease can produce bilateral hallucinations, it is very difficult to show this, even if true, from published reports.

He does not discuss the crucial question of whether ear disease without central disinhibition or neurological or psychiatric disease is a sufficient cause of auditory hallucinations. If so, concomitant brain disease may be a red herring. Dr Berrios claims a link between right hemisphere lesions and musical hallucinations, unsupported in recent well studied cases (Cambier et al, 1987; Shapiro et al, 1991). This makes it even more unfortunate that he had no comparable matching group of non-psychotic patients with verbal hallucinations linkable to left hemisphere lesions. 
Dr Berrios ignores the literature on brainstem auditory hallucinosis. Cambier et al (1987) reported four such cases with typical religious/patriotic/ music-hall themes, attributed to auditory deafferentation. In fact, the site of lesion was ambiguous, although all had brainstem lesions and none cortical ones. All were bilaterally deaf and auditory nerve lesions could not be excluded audiologically; the brainstem neurological symptoms resolved but not the deafness. A further case with an apparently pure brainstem lesion had primitive auditory hallucinations during one night of insomnia.

I conclude that Dr Berrios's review (and mine for that matter) were both incomplete, and it is unfortunate, but predictable, that his review is now taken as the last word up to 1989 on musical and related hallucinations.

32 Love Walk

A. G. GORDON

London SE5 8AD

\section{References}

Cambier, J., Decroix, J. P. \& Masson, C. (1987) Hallucinose auditive dans les lesions du tronc cerebral. Revue Neurologique, 143 255-262.

GoRDON, A. G. (1987) Unilateral otopathic hallucinations-a review. Acta Psychiatrica Scandinavica. 75, 664-668.

- (1988) Unilateral auditory hallucinations. British Journal of Psychiatry, 153, 263-264.

Sha piro. C. M., Kasem, H. \& Tewari, S. (1991). My music-a case of musical reminiscence diagnosed courtesy of the BBC. Journal of Neurology, Neurosurgery \& Psychiatry, 54, 88-89.

\section{Failure to convulse with ECT}

SIR: Failure to convulse with electroconvulsive therapy (ECT) has been estimated to occur on up to $21 \%$ of occasions that the electrical stimulus is administered (Pettinati \& Nilsen, 1985). The topic has received much attention in recent years in the British Journal of Psychiatry (e.g. Journal, January 1988, 152, 134-136; Journal, April 1988, 152, 571; Journal, May 1988, 152, 712-713). Following my own correspondence (Journal, May 1990, 156, 747-748) I have been made aware of the Royal College's recommendations (Freeman et al, 1989) concerning what should be done if there is no observable seizure during ECT. While welcoming these guidelines, it is interesting to contrast them with those of the American Psychiatric Association (1990). The two regimens differ, for example, in:

(a) the number of restimulations that can be applied

(b) the requirement that a designated time elapse between restimulations

(c) the magnitude of the parameters of the repeat stimuli.
Although unsafe practice cannot be inferred from diverse practice, there clearly remains a need for a more uniform protocol, validated by empirical data. It is sobering to recall that when ECT was first attempted, in mid-April 1938, the initial stimulus of 70 volts for 0.2 seconds was unintentionally subconvulsive. Cerletti remembered (Cerletti, 1950) that a voluble discussion then broke out among the spectators, who included Bini, Longhi, Accornero, Kalinowski and Fleischer. Most objected to a further shock. The patient himself protested: "Non una seconda! Mortifera!" (Not another! It will kill me!). With some trepidation Cerletti decided to administer another stimulus of 110 volts for 0.5 seconds and the patient convulsed. It is a sad reflection on our discipline and, in this era of quality assurance, perhaps unsatisfactory that the protocols that have been devised to allay Cerletti's trepidation continue to lack empirical support and are often conflicting.

The Northside Clinic

GARRY WALTER

Sydney

Australia

\section{References}

Cerlettr, U. (1950) Old and new information about electroshock. American Journal of Pschiatry, 107, 93-94.

Pettinati, H. M. \& Nilson, S. (1985) Missed and brief seizures during ECT: differential response between unilateral and bilateral electrode placement. Biological Psychiatry. 20, 506-514.

Freeman, C., Crammer, J. L., Deakin, J. F. W. et al (1989) (ECT Sub-committee of the Research Committee of the Royal College of Psychiatrists). The Practical Administration of Electroconvulsive Therapy. London: Gaskell.

American Psychiatric Association (1990) The Practice of Electroconvulsive Therapy: Recommendations for Treatment, Training and Privileging. (Task Force Report). Washington, DC: APA.

\section{Anorexia nervosa in the elderly}

SIR: I read the report of "anorexia nervosa" in a 73-year-old woman by Cosford \& Arnold (Journal. February 1991, 158, 286-287) with interest. This lady suffered her (first?) episode of marked weight loss, food avoidance (why?), fear of weight gain and amenorrhoea after a loss at the age of 23 years. She received an unstated treatment over nine months as an in-patient. Fifty years later she "suffered a relapse, with severe weight loss, a distorted body image and a fear of becoming fat". This time she was put on a strict diet and was discharged after five months. This makes 14 months in-patient treatment. Is there an alternative explanation? Was it an atypical affective disorder. Did the passage of months (and the affective episode) provide the 'cure'?

Bernstein(1972) described the successful treatment of a 94-year-old woman with "anorexia nervosa" 